\title{
INTERMITTENT MANDATORY VENTILATION AND CONTROLLED MECHANICAL VENTILATION WITHOUT POSITIVE END-EXPIRATORY PRESSURE FOLLOWING CARDIO-PULMONARY BYPASS
}

\author{
Robert N. SLAden and LEONARD C. Jenkins
}

Pulmonary DYSFUnCTION after cardiac surgery is well-known. In a study at St Paul's Hospital, Vancouver, Turnbull, et al' found that the incidence of pulmonary atelectasis after cardiopulmonary bypass was more than 50 per cent. The alveolar-arterial oxygen gradient $\left(\mathrm{A}-\mathrm{aDo}_{2}\right)_{\text {, }}$ which correlated well with clinical and X-ray findings, deteriorated markedly after operation, and took as long as seven days to return to normal. Mechanical ventilation in this group of patients was provided for not longer than four hours after operation. In recent years the optimum duration of ventilation following cardio-pulmonary bypass has been disputed. In many centres, routine overnight mechanical ventilatory support is preferred, ${ }^{2,3}$ Midell and Skinner ${ }^{4}$ oppose this view. At the time of the present study, this problem had not been resolved in the Willow Chest Cardiothoracic Unit (WCTU) of the Vancouver General Hospital.

Since 1972 another mode of ventilatory support, intermittent mandatory ventilation (IMV), has come into prominence. Review of the literature reveals a single study of the use of IMV following cardio-pulmonary bypass: a series of 21 patients by Downs and Modell, 5.6 two of the pioneers of this technique. They found that functional residual capacity (FRC) was improved after operation only when IMV was used with positive end-expiratory pressure (PEEP). Their patients were followed for the first eight hours after operation. The results lend weight to the impression that many of the advantages claimed for IMV exist through its facilitation of the use of PEEP.

\section{Aims}

The aims of the present study were threefold: First, to study the effect of overnight ventilation on the incidence of a pulmonary atelectasis

Robert N. Sladen, M.B., M.R.C.P., Resident, Leonard C. Jenkins, M.D., C.M., F.R.C.P.(C), Professor and Head, Department of Anaesthesiology, University of British Columbia, Vancouver General Hospital, Vancouver, B.C. , Canada, V5Z IM9.

Canad. Anaesth. Soc. J., vol. 25, no. 3, May 1978 as demonstrated by chest $\mathrm{X}$-ray and $\mathrm{A}-\mathrm{aDo} \mathrm{O}_{2}$. It was hoped that the findings in this respect would help clarify the approach to post-operative mechanical ventilation at the WCTU in the Vancouver General Hospital.

Second, to compare and contrast the effects of controlled mechanical ventilation (CMV) with those of IMV when the latter was divorced from its habitual partner, PEEP. This was designed to determine whether IMV is of benefit through its facilitation of PEEP.

Third, to set up a protocol of study for further research on the use of IMV in the WCTU in particular, and as a follow-up to the present series, the use of IMV with PEEP.

\section{MeTHOD}

Eighteen male patients scheduled for saphenous vein coronary artery bypass grafting were studied. Careful clinical assessment, electrocardiogram, chest X-ray and arterial blood gases were done pre-operatively in order to exclude patients with cardiac failure or overt obstructive lung disease, so as to make the group as uniform as possible. Alveolar-arterial oxygen gradients were measured as follows: the patients breathed 100 per cent oxygen for 20 minutes through a non-rebreathing circuit consisting of bubble humidifier, 3-litre reservoir bag, Rubens valve, mouthpiece and noseclip. Arterial blood gases were then taken and the $\mathrm{A}-\mathrm{aDO}_{2}$ was calculated using the alveolar air equation.* All measurements were made with the patient in the supine position.

A standard anaesthetic was given. Premedication consisted of oral diazepam followed by intramuscular morphine and atropine. Patients were induced with intravenous diazepam, thiopentone and succinylcholine together with an on-going infusion of meperidine. Anaesthesia was maintained with a mixture of nitrous oxide and oxygen, halothane, meperidine infusion and

$\left.{ }^{*} \mathrm{~A}-\mathrm{aDO} \mathrm{O}_{2}=\mathrm{PI}_{\mathrm{O}_{2}}-\mathrm{PH}_{2} \mathrm{O}-\mathrm{Pa}_{\mathrm{CO}_{2}} / \mathrm{R}\right)-\mathrm{Pa}_{\mathrm{O}_{2}}$ torr. where $\mathrm{Pl}_{\mathrm{O}_{2}}=$ atmospheric pressure, $\mathrm{PH}_{2} \mathrm{O}=$ water vitpour pressure $R=0.85$ (after Turnbull ${ }^{1}$ ). 


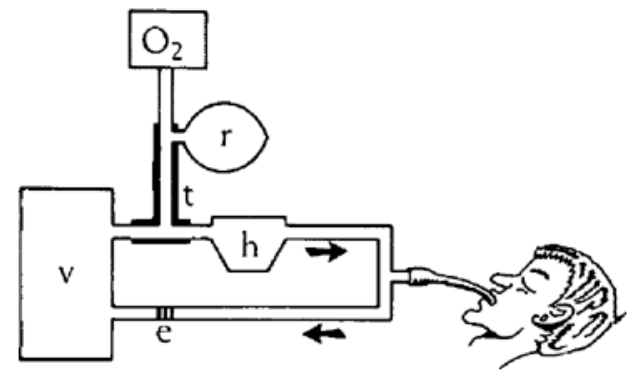

Figure 1, Intermittent Mandatory Ventilation (IMV) circuit. For explanation see text.

increments of $D$-tubocurarine. Bypass was provided by the Travenol bubble oxygenator with halothane added through a vaporizer. This anaesthetic regime is one used regularly in the WCTU; in this way it was hoped that results would be pertinent to current practice there.

At the end of operation patients were transferred to the post-anaesthetic room (PAR) where they were attached to the Bennett MA-1 volume ventilator. Tidal volumes of $13 \mathrm{ml} / \mathrm{kg}$ were provided. Rate was adjusted to give a minute ventilation of $120 \mathrm{ml} / \mathrm{kg} / \mathrm{min}$. Sedation in the form of intravenous morphine and diazepam was titrated to control pain and ventilation. PEEP was not applied and muscle relaxants were avoided.

At this stage the patients were allocated to either the CMV or IMV group. Assignments had been made in a random fashion at the preoperative visit.

IMV was provided by a modification of the standard Bennett MA-1 ventilator, illustrated in Figure 1. A spontaneous breathing circuit consists of wall oxygen through a Veriflo air mixer $\left(\mathrm{O}_{2}\right)$, reservoir bag $(r)$ and $t$-piece with one-way valve $(t)$. This is inserted into the inspiratory limb between the ventilator and the cascade humidifier (h). A slow-rate circuit card and dial adapter allows ventilator rates as low as one per minute to be delivered.

Patients in the CMV group were ventilated overnight. Sedation was given for pain and to allow mechanical control of ventilation. Weaning was commenced next morning. The patients were extubated when the usual criteria used in the WCTU were met. These criteria are (1) tidal volume more than $5 \mathrm{ml} / \mathrm{kg}$, (2) forced vital capacity more than $10 \mathrm{ml} / \mathrm{kg}$, (3) ability to tolerate the t-piece for 30 minutes without change in vital signs, hypoxia or hypercarbia.

Patients in the IMV group had their ventilation controlled for the first four hours in the post- anaesthesia recovery room. Weaning was then commenced. Every four hours the ventilator rate was reduced by one or two breaths per minute provided that (1) arterial $\mathrm{PCO}_{z}$ remained less than $5.99 \mathrm{kPa}(45$ torr) or arterial $\mathrm{pH}$ greater than 7.35 and (2) there was no tachypnoea. If the patient could tolerate IMV rates of one to two per minute, he was considered to be ready for extubation.

After extubation, both groups were given humidified oxygen by mask.

Chest X-ray and $\mathrm{A}-\mathrm{aDO}_{2}$ were done on each patient six times: (1) At the pre-operative visit; (2) on admission to the post-anaesthetic recovery room; thereafter, at approximately eight-hour intervals: (3) and (4) on mechanical ventilation; (5) and (6) breathing spontaneously. Total duration of post-operative follow-up was $\mathbf{4 4}$ hours.

Data were analysed by Student's paired t-test, except for the chest $X$-rays, which were analysed by the method of binomial variables (random blocks).

\section{Results}

\section{(1) Clinical data}

There was no significant difference between the two groups of patients when they were matched for the following data: age, weight, preoperative $\mathrm{Pa}_{2}$ and $\mathrm{A}-\mathrm{aDO}_{2}$, history of smoking, previous myocardial infarction, anaesthesia time, crystalloid load, change in haematocrit, blood balance and urine output. Cardiopulmonary bypass time was marginally longer in the IMV group ( $\mathrm{p}<0.05, \mathrm{p}>0.02)$.

It was noted that in 50 per cent of cases, the indication for surgery was angiographic disease rather than incapacitating angina pectoris. Half the total group had had myocardial infarction previously, though only one within six months of anaesthesia. Sixty per cent of patients were on propranolol, which was discontinued the day before operation. Half the patients were smoking more than 20 cigarettes per day six months prior to operation.

Mean total intra-operative crystalloid load was approximately five litres, or one litre per hour of anaesthesia.

These data are tabulated in Tables I and II.

\section{(2) Alveolar-arterial oxygen gradients}

There was a marked increase in $\mathrm{A}-\mathrm{aDO}_{2}$ after operation in both groups of patients, as illustrated in Figure 2. Quantitatively it was similar to that reported by Tumbull, et al., ${ }^{1}$ in 1974. 
TABLE I

Comparison of Patient Groups

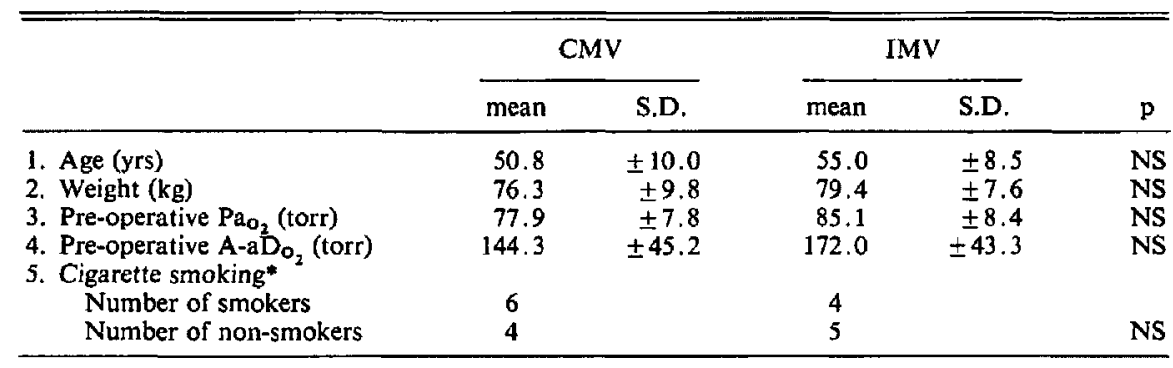

NS $=$ not significantly different $(p>0.05)$.

-Refers to smoking of 20 or more cigarettes/day less than six months before operation. Analysed by method of chi-squared.

TABLE If

Comparison of Intra-Operative Data

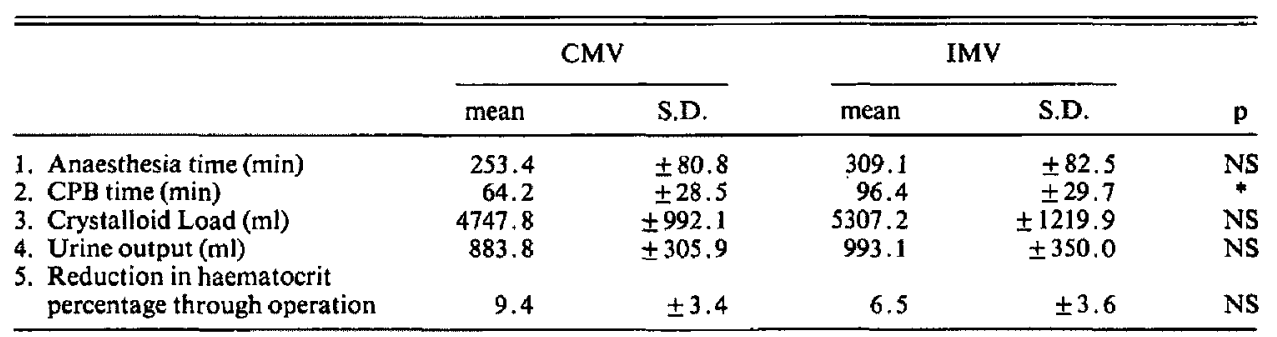

$\mathrm{CPB}=$ Cardiopulmonary bypass, NS $=$ not significantly different $(\mathrm{p}>0.05)$.

${ }^{*} p<0.05$, $>0.02$, marginally significant difference.

In the CMV group (solid line), the $\mathrm{A}-\mathrm{aDO}_{2}$ decreased slightly after eight hours of ventilation, but increased overnight. In the IMV group (dashed line), the $\mathrm{A}-\mathrm{aDo}_{2}$ showed progressive but moderate increase overnight. Overall, there was no significant difference in the effect on $\mathrm{A}-\mathrm{aDO}_{2}$

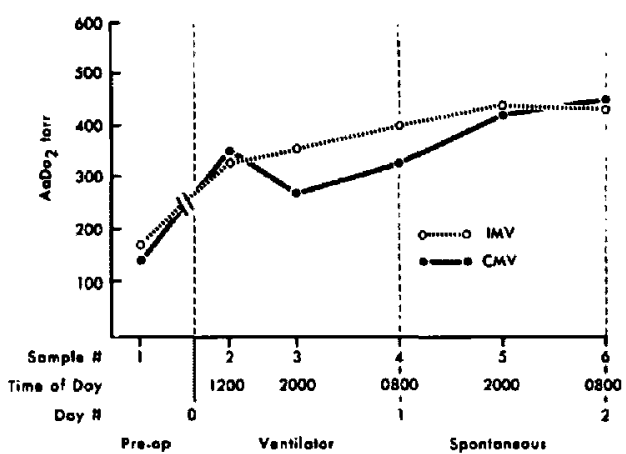

Figure 2. Alveolar-arterial oxygen gradients measured after breathing 100 per cent oxygen for $20 \mathrm{~min}$ utes. Sample 1, pre-operative: samples $2,3,4$, on mechanical ventilation; samples 5,6 , during spontaneous ventilation. whether CMV or IMV was used ( $p>0.05)$. After extubation, $\mathrm{A}-\mathrm{aDO}_{2}$ values for both groups approached similar levels.

It thus appears that overnight ventilation by either mode did not prevent or reverse postoperative increase in $\mathrm{A} \cdot \mathrm{aDo}_{2}$ (and, by inference, intra-pulmonary shunting). Indeed, it continued to increase despite the ventilation. After extubation and for the next eight hours, patients had similar results regardless of the previous mode of ventilation.

\section{(3) Chest $X$-rays}

The chest $X$-rays were examined in a singleblind fashion by a staff radiologist and graded on a scale of 0 to 4 for degree of atelectasis.

In Figure 3, the degree of atelectasis is represented by the following scoring system on the horizontal scale: $0=$ normal lung; 1 = nonspecific change $; 2=$ subsegmental atelectasis; 3 = segmental atelectasis; $4=$ lobar atelectasis. The vertical bars are subdivided to represent day 0,1 or 2 .

Statistical analysis confirms the graphic im- 


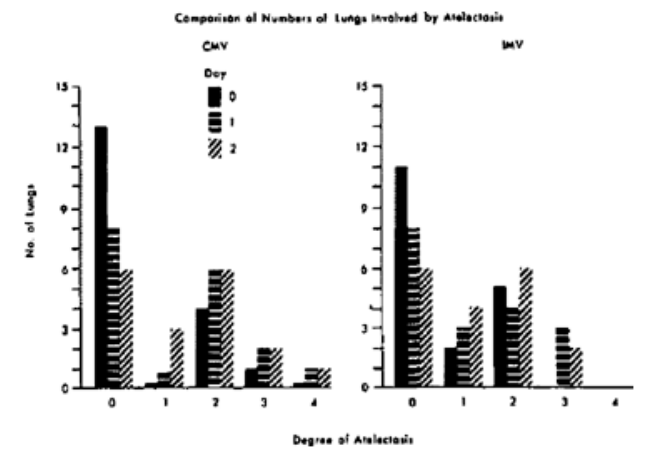

Figure 3. Comparison of incidence and degree of atelectasis between CMV group (left) and IMV group (right). Degree of atelectasis within each group increases in severity from left to right on the abscissa (see text for explanation of scoring code); the height on the ordinate indicates the number of lungs involved by that degree of atelectasis. Solid line $=$ day 0 ; horizontal hatching $=$ day 1 ; diagonal hatching $=$ day 3 . The graphs illustrate that the number of lungs involved by atelectasis increases on successive days; however, there is no significant difference in the over-all pattern between the two groups.

pression that there was no difference in the incidence of atelectasis between the CMV group and the IMV group $(p>0.05)$. Examination of the vertical bars reveals that there is a "shift to the right" with respect to the degree of atelectasis over successive days. Thus, as with the $\mathrm{A}-\mathrm{aDO}_{2}$, there is shown to be an increase in atelectasis despite overnight ventilation.

When the lungs of both groups are combined, the dominant involvement of the left side is revealed $(p<0.01)$ (Figure 4 ).

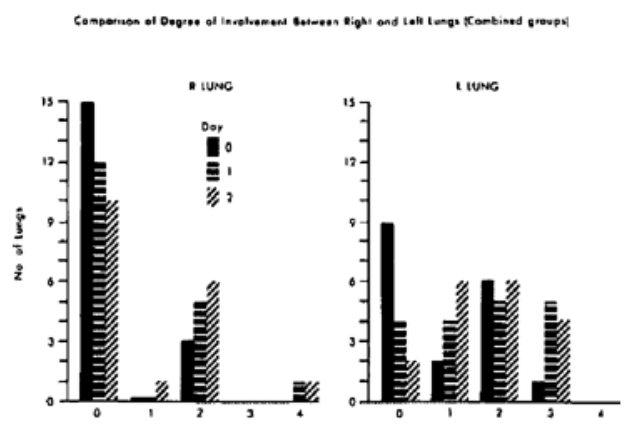

Figure 4. Comparison of incidence and degree of atelectasis between right lung (left) and left lung (right) when the two groups are combined. Explanation of the graphs is the same as for Figure 3 . Here they demonstrate that when the two groups are combined, there is a significantly greater degree of involvement by atelectasis in the left lung than in the right lung.
In summary, the chest $\mathrm{X}$-rays demonstrated that the overall incidence of post-operative atelectasis - 60 per cent of all cases - was no different whether CMV or IMV without PEEP was provided overnight after operation. Most atelectasis was left-sided and subsegmental.

The most noteworthy fact to emerge from a study of the chest X-rays, however, appears to be that of the eleven patients who developed atelectasis, eight (70 per cent) developed it during anaesthesia. Once established, atelectasis was not reversed or improved by the modes of mechanical ventilation used in this study.

\section{Discussion}

The results obtained in this study give rise to three questions regarding post-operative ventilation after cardio-pulmonary bypass:

First, if IMV without PEEP is no better than CMV, why use it at all? Second, why did the greatest proportion of atelectasis develop during anaesthesia? Third, if the mode of ventilation used overnight in this study does not prevent atelectasis or reverse that already established, what will?

\section{(1) Why intermittent mandatory ventilation?}

IMV was first introduced into adult medicine in an attempt to solve the problem of difficult weaning from the ventilator. ${ }^{7-13}$ Its proponents claimed that weaning with the new modality is more precise, simple and physiological than orthodox t-piece weaning. Klein ${ }^{14}$ depicted the difference between the two methods succinctly: in t-piece weaning, mechanical ventilatory support is withdrawn in a staccato fashion, as an all-ornone phenomenon; in IMV weaning, it is withdrawn in a smooth, graded manner. The patient is not exposed to the possibility of sudden hypoxia or hypercarbia. Two groups of patients present weaning difficulties: those with chronic obstructive lung disease ${ }^{15}$ and those on prolonged mechanical ventilation. Neither of these problems existed in this series; in fact, the patients were pre-selected to be in relatively good status in order to achieve uniformity. Difficulty in weaning was not expected and, indeed, was not found. No conclusions may be drawn about IMV weaning from this study.

The second advantage ascribed to IMV is that, when compared to continuous mechanical ventilation, the mean intrathoracic pressure that results is at a lower level. With every spontaneous breath that occurs during IMV, the mean in- 
trathoracic pressure is reduced below atmospheric. ${ }^{14.16}$ When PEEP is applied, the overall effect on this pressure is thus modified. Higher levels of PEEP are less likely to reduce cardiac output and renal perfusion. In patients whose cardiac reserve is critical, any intermittent positive pressure ventilation, with or without PEEP, may cause the cardiac output to fall precipitously unless extremely careful haemodynamic support is provided.

IMV would therefore seem to be an attractive modality for use with the cardiac patient. Bryan-Brown ${ }^{17}$ has stated that it is the ventilatory mode of choice for patients in cardiac failure.

Several advantages of the use of IMV are necessarily based on subjective impressions of the staff working with the mode and do not lend themselves to tabulation or statistical analysis. For example, it is claimed that less sedation is required for tolerance of IMV than for CMV because the patient is allowed to breathe spontaneously between machine cycles. In this study, narcotic requirements during the first 12 hours in the post-anaesthetic recovery room were almost identical in both groups (mean total worphine: CMV $32 \mathrm{mg}, \mathrm{SD} \mp 10.2$; IMV $31.5 \mathrm{mg}, \mathrm{SD} \mp 9.0$ ). However, patients on IMV required substantially less diazepam than those on CMV (mean total diazepam: CMV $21 \mathrm{mg}, \mathrm{SD} \mp 8.6$; IMV $13 \mathrm{mg}, \mathrm{SD}$ $\mp 8.6$ ). Although this difference should not be analysed statisticaliy, it may indicate a clinically valid trend.

In summary, this study demonstrated that insofar as the prevention of post-operative atelectasis is concerned, IMV without PEEP is no better than CMV. However, in view of the benefits found in patients with obstructive lung disease, weaning difficulty and especially cardiovascular impairment, further investigation of the use of IMV with PEEP is justified. A clinical impression gained in this study is that although patients still require adequate narcosis for pain relief and tolerance of tracheal intubation, the requirement for tranquillization (and possible "hangover" after extubation) is diminished.

(2) Why does so much atelectasis occur during anaesthesia?

In an excellent description of the evolution of atelectasis, Wilson and Pontoppidan ${ }^{18}$ discussed the many factors that interact in causing collapse of lung during anaesthesia and mechanical ventilation.

Any monotonous pattern of ventilation will lead to progressive miliary alelectasis and small airway closure. ${ }^{19-22}$ Positive pressure manoeuvres such as large tidal volumes, intermittent sighing and PEEP may prevent this, but alt have the potential of reducing cardiac output. Increased pulmonary extravascular water through excessive crystalloid loading (which increases hydrostatic pressure and reduces colloid osmotic pressure) pred isposes to airway closure, alveolar collapse and pulmonary infection, ${ }^{23,24}$ Surfactant activity is deficient amongst smokers. Its production may cease with the abnormal lung volumes and regional pulmonary ischaemia that occur during cardiopulmonary bypass. ${ }^{25}$

Blood may enter the pleural cavity if it is opened during operation. During bypass the heart rests on the immobile left lower lobe. ${ }^{26}$ Secretions, more profuse in smokers, are imperfectly cleared during tracheal intubation and mechanical ventilation. Blind bronchial suctioning may make matters worse by damaging tracheal mucosa and causing secretions to "dam up". The left main bronchus is more difficult to enter and is more likely to be damaged. This may explain the greater incidence of left-sided atelectasis. ${ }^{26}$

Elderly patients have decreased lung elasticity and increased closing volumes. Functional residual capacity (FRC) is reduced in obese patients. Passive ventilation of the paralysed anaesthetized patient causes diaphragmatic displacement with reduced FRC and ventilationperfusion mismatching. ${ }^{27}$

All these factors may act in a generalized or localized manner to cause atelectasis during anaesthesia. The pre-operative and intraoperative management of the patient is thus of equal importance in the prevention of postoperative pulmonary complications and it is to this area that we should be directing more attention.

\section{(3) What will prevent atelectasis?}

From the discussion above and from the results in this study, one may conclude that reduction in intra-operative atelectasis will reduce morbidity in the post-operative period. A clinical approach based on known physiological principles should be applied.

For example, all patients should have basic pulmonary function tests such as FVC and FEV, to serve as a guide to possible post-operative pulmonary complications and as a baseline for response to physiotherapy. Patients who have profuse overnight secretions should have their operations deferred until the afternoon so that they may be "cleared out" in the morning. ${ }^{28}$ 
Strict criteria for cessation of smoking and reduction of obesity before operation should be applied.

During operation special attention should be given to lung inflation. A study at this centre demonstrated that the application of $10 \mathrm{~cm} \mathrm{H}_{2} \mathrm{O}$ PEEP to the passively inflated lungs during bypass did not reduce the incidence of atelectasis after operation (Turnbull, unpublished observations). Careful re-inflation of the lungs, and especially the left lower lobe, should be performed after bypass, perhaps supplemented by intermittent sighing during closure. Fibreoptic bronchoscopy should replace blind bronchial suctioning, ${ }^{26,29}$ and could perhaps be performed at the end of anaesthesia. Excessive crystalloid administration can be avoided by the use of albumen when expansion of intra-vascular volume is desired.

Certainly this study has shown that in a preselected relatively low-risk group of patients, overnight ventilation with CMV or IMV without PEEP is of little benefit in the management or avoidance of post-operative atelectasis. On the other hand, premature extubation in the absence of adequate cough or ventilatory ability, or in the presence of severe pain or cardiac decompensation, is in itself dangerous. Indeed, in many cases mechanical ventilation is essential to reduce the work of breathing in the sick cardiac patient. It would seem, therefore, that ventilation should be tailored to meet the demands of the particular situation. To this end one can hardly overemphasize the need for close co-operation and discussion between surgeon and anaesthetist in the post-anaesthetic recovery room.

\section{SUMMARY}

In a group of 18 male patients undergoing coronary artery bypass grafting with cardiopulmonary bypass, the overall incidence of post-operative atelectasis was 60 per cent. Nearly three-quarters occurred during anaesthesia. After operation there was no difference whether CMV or IMV without PEEP was provided overnight. Atelectasis already present did not improve and further atelectasis occurred.

A role for IMV is not excluded, since it facilitates the use of PEEP. Many factors operate and interact to provoke atelectasis during anaesthesta, which increases post-operative morbidity. Many of these factors are preventible or reversible if their physiological basis is understood.

Optimal post-operative ventilation should be tailored to the needs of the individual patient and demands close co-operation between anaesthetist and surgeon.

\section{RÉSUMÉ}

L'incidence d'atélectasie post-opératoire a été de 60 pour cent chez 18 patients ayant subi un pontage aorto-coronarien à coeurouvert. Troisquart de ces complications sont survenues au cour's de l'anesthésie. On n'a pas noté de différence en utilisant une ventilation obligatoire intermittente (IMV) sans peep au cours de la première nuit au lieu de la ventilation contrôlée ordinaire. Non seulement l'atélectasie déjà présente à l'arrivée aux soins intensifs ne s'améliorait pas, mais au contraire il s'en produisait davantage.

L'emploi de la ventilation obligatoire intermittente peut quand même présenter des avantages car elle diminue les effets de la peep sur le débit cardiaque.

De nombreux facteurs contribuent à la production d'atélectasie durant l'anesthésie, ce qui augmente la morbidité post-opératoire. Beaucoup de ceux-ci peuvent être prévenus ou corrigés si leur physiopathologie est comprise. L'assistance respiratoire post-opératoire devrait être choisie en fonction d'un patient donné, ce qui implique une collaboration étroite entre le chirurgien et l'anesthésiste.

\section{ACKNOWLEDGEMENTS}

We wish to thank Dr. J.P. Munroe for his constant guidance and encouragement. We are indebted to the nurses and therapists of the postanaesthetic recovery room of the Willow Chest Cardiothoracic Unit at the Vancouver General Hospital; without their enthusiastic co-operation this study would have been impracticable. Finally, we are most grateful to Dr. J. Clement for evaluation of the chest X-rays and to Dr. M. Schulzer of the Department of Mathematics of the University of British Columbia for statistical analysis.

\section{REFERENCES}

1. Turnbull, K.W., MiYagishima, R.T., \& Ge REIN, A.N. Pulmonary complications and cardiopulmonary bypass: a clinical study in adults. Can. Anaesth. Soc. J. $21: 181$ (1974).

2. Estafanous, F.G. Respiratory care following open-heart surgery. Surg. Clin. N. Amer. 55: 5: 1299 (1975).

3. Campbell, G.S. Respiratory failure in surgica! patients. Current Problems in Surgery 13: 2: 1 (1976).

4. Midell, A.I., Skinner, D.B., De Boer, A., \& Bermudez, G. A review of pulmonary problems 
following valve replacement in 100 consecutive patients. Ann. Thor. Surg. 18: 219 (1974).

5. Downs, J.B., Mitchell, L.A., Dannemiller, F.J., \& Stanford, W. Modification of airway closure and pulmonary gas exchange following cardiac surgery (abstract). Critical Care Med. $3: 41$ (1975).

6. Downs, J.B. \& Mitchell, L.A. Pulmonary effects of ventilatory pattern following cardiopulmonary bypass. Critical Care Med. 4: 295 (1976).

7. Margand, P.M.S. \& Chodoff, P. IMV: an alternative weaning technic: a case report. Anesth. and Analg. 54: 41 (1975).

8. Downs, J.B., Klein, E.F., Desautels, D., MODELL, J.H., \& KIRBY, R.R. IMV: a new approach to weaning patients from mechanical ventilators. Chest $64: 331$ (1973).

9. KIRBY, R.R. A new pediatric volume ventilator. Anesth. and Analg. 50: 533 (1971).

10. Kiray, R.R., Rorison, E., Schulz, J., \& DE LEMos, R.A. Continuous flow ventilation as an alternative to assisted and controlled ventilation in infants. Anesth. and Analg. 5/: 871 (1972).

11. Downs, J.B., Perkins, H.M., \& Modell, J.H. Intermittent mandatory ventilation: an evaluation. Arch. Surg. 109: 519 (1974).

12. Feeley, T.W. \& Hedley-Whyte, J Current concepts: weaning from controlled ventilation and oxygen. New Eng. J. Med. 292: 903 (1975).

13. KIRBY, R.R. Is IMV a satisfactory alternative to assisted and controlled ventilation. Lecture \#207b. Annual Refresher Course Lectures. A.S.A. 1 (1975).

14. KLEIN, E.F. Weaning from mechanical breathing with IMV. Arch. Surg. 1 10: 345 (1975).

15. Downs, 3.B., Block, A.J., \& Vennum, K.B. Intermittent mandatory ventilation in the treatment of patients with chronic obstructive pulmonary disease. Anesth. and Analg. 53: 437 (1974).

16. Kirby, R.R., Perry, J.C., Calderwood, H.W. RU1z, B.D.. \& LeDERMAN, D.S. Cardiorespiratory effects of high positive end-expiratory pressure. Anesthesiology 43: 533 (1975).

17. Bryan-Brown, C.W. Guidelines for ventilatory management in patients in heart failure. Abstracts:
I4th Symposium on Critical Care Medicine: 21 (1976).

18. Wilson, R.S. \& Pontoppidan, H. Acute respiratory failure: diagnostic and therapeutic criteria. Critical Care Med. 2: 293 (1974).

19. Laver, M.B., Morgan, J., Bendixen, H.H., \& RADFORD, E.P. Lung volume, compliance and arterial oxygen tensions during controlled ventilation. J. Appl. Phys. 19: 725 (1964).

20. Hedley-Whyte, J., Laver, M.B., \& Bendixen, H.H. Effect of change in tidal ventilation on physiological shunting. Amer. J. Physiol. 206: 891 (1964).

21. Bartlett, R.H., Krop, P., Hanson, E.L., \& MOORE, F.D. Physiology of yawning and its application to post-operative care. Surg. Forum $2 l: 222$ (1970).

22. Bartlett, R.H., Gazzaniga, A.B., \& GeRAGHTY, T.R. Respiratory manoeuvers to prevent post-operative pulmonary complications. J. Amer. Med. Assoc. 224: 1017 (1973).

23. La Force, F.M., Mullane, J.F., Boemme, R.F. KELLY, W.J., \& HUBER, G.L. The effect of pulmonary edema on antibacterial defenses of the lung. J. Lab. Clin. Med. 82: 634 (1973).

24. ROTH, E., LAX, L.C. \& Maloney, J.V. Ringer's lactate solution and extracellular fluid volume in the surgical patient: a critical analysis. Ann. Surg. 160: 149 (1969).

25. Comroe, J.H. Physiology of Respiration, 2 nd ed. Chicago: Year Book (1974).

26. Lindholm, C., Ollman, B., Snyder, J., MilLEN, E., \& GRENVIK, A. Flexible fieroptic bronchoscopy in critical care medicine. Critical Care Med. 2: 250 (1974)

27. Froese, A.B. \& Bryan, C.B. Effects of anaesthe sia and paralysis on diaphragmatic mechunics in man. Anesthesiology 4I: 242 (1974).

28. VeITH, F.J. \& RoeIo, A.G. Evaluation of respiratory function in surgical patients; importance of pre-operative preparation and prediction of putmonary complications. Surgery 45: 905 (1959).

29. Milledge, J.S. Therapeutic fibreoptic bronchoscopy in intensive care. Brit. Med. J. 2: 1427 (1976). 